PROCEEDINGS OF THE

AMERICAN MATHEMATICAL SOCIETY

Volume 138, Number 2, February 2010, Pages 743-751

S 0002-9939(09)10140-5

Article electronically published on October 1, 2009

\title{
CIRCLE IMMERSIONS THAT CAN BE DIVIDED INTO TWO ARC EMBEDDINGS
}

\author{
KOUKI TANIYAMA \\ (Communicated by Alexander N. Dranishnikov)
}

\begin{abstract}
We give a complete characterization of a circle immersion that can be divided into two arc embeddings in terms of its chord diagram.
\end{abstract}

\section{INTRODUCTION}

Let $\mathbb{S}^{1}$ be the unit circle. Let $X$ be a set and $f: \mathbb{S}^{1} \rightarrow X$ a map. Let $n$ be a natural number greater than one. Suppose that there are $n$ subspaces $I_{1}, \cdots, I_{n}$ of $\mathbb{S}^{1}$ with the following properties:

(1) Each $I_{i}$ is homeomorphic to a closed interval.

(2) $\mathbb{S}^{1}=I_{1} \cup \cdots \cup I_{n}$.

(3) The restriction map $\left.f\right|_{I_{i}}: I_{i} \rightarrow X$ is injective for each $i$.

Then we say that $f$ can be divided into $n$ arc embeddings. We define the arc number of $f$, denoted by $\operatorname{arc}(f)$, to be the smallest such $n$ except for the case that $f$ itself is injective. If $f$ itself is injective, then we define $\operatorname{arc}(f)=1$. If $f$ cannot be divided into $n$ arc embeddings for any natural number $n$, then we define $\operatorname{arc}(f)=\infty$.

Note that if $f$ can be divided into $n$ arc embeddings, then there exist $n$ subspaces $I_{1}, \cdots, I_{n}$ of $\mathbb{S}^{1}$ with (1), (2) and (3) above together with the following additional condition.

(4) $I_{i} \cap I_{j}=\partial I_{i} \cap \partial I_{j}$ for each $i$ and $j$ with $1 \leq i<j \leq n$.

Namely, we may assume that $\mathbb{S}^{1}$ is covered by mutually interior disjoint $n$ simple $\operatorname{arcs} I_{1}, \cdots, I_{n}$.

Let $\mathrm{S}(f)=\left\{x \in \mathbb{S}^{1} \mid f^{-1}(f(x))\right.$ is not a singleton $\}$ and $\mathrm{s}(f)=f(\mathrm{~S}(f))$. We say that a map $f: \mathbb{S}^{1} \rightarrow X$ has finite multiplicity if $\mathrm{S}(f)$ is a finite subset of $\mathbb{S}^{1}$. From now on we restrict our attention to maps that have finite multiplicity. The purpose of this paper is to give a characterization of a map $f: \mathbb{S}^{1} \rightarrow X$ with $\operatorname{arc}(f)=2$. By $|Y|$ we denote the cardinality of a set $Y$. Let $m(f)$ be the maximum of $\left|f^{-1}(y)\right|$, where $y$ varies over all points of $X$. It is clear that $\operatorname{arc}(f) \geq m(f)$. Thus we further restrict our attention to a map $f: \mathbb{S}^{1} \rightarrow X$ whose multiple points are only finitely many double points; namely $f$ has finite multiplicity and $m(f) \leq 2$. Then we have $|\mathrm{S}(f)|=2 m$ for some non-negative integer $m$. Then the crossing number of $f$, denoted by $c(f)$, is defined to be $m$.

Received by the editors February 9, 2009, and, in revised form, April 2, 2009.

2000 Mathematics Subject Classification. Primary 57M99; Secondary 57M25, 57M27.

Key words and phrases. Circle immersion, chord diagram, plane curve, knot projection.

The author was partially supported by Grant-in-Aid for Scientific Research (C) (No. 18540101), Japan Society for the Promotion of Science.

(c)2009 American Mathematical Society 743

Reverts to public domain 28 years from publication 
Let $m$ be a natural number. An $m$-chord diagram on $\mathbb{S}^{1}$ is a pair $\mathcal{C}=(P, \varphi)$, where $P$ is a subset of $\mathbb{S}^{1}$ that contains exactly $2 m$ points and $\varphi$ is a fixed point free involution on $P$. A chord $c$ of $\mathcal{C}$ is an unordered pair of points $(x, \varphi(x))=(\varphi(x), x)$, where $x$ is a point in $P$. Let $\sim_{\mathcal{C}}$ be the equivalence relation on $\mathbb{S}^{1}$ generated by $x \sim_{\mathcal{C}} \varphi(x)$ for every $x \in P$. Let $\mathbb{S}^{1} / \sim_{\mathcal{C}}$ be the quotient set and $f_{\mathcal{C}}: \mathbb{S}^{1} \rightarrow \mathbb{S}^{1} / \sim_{\mathcal{C}}$ the quotient map. We call $f_{\mathcal{C}}$ the associated map of $\mathcal{C}$. Then the arc number of $\mathcal{C}$, denoted by $\operatorname{arc}(\mathcal{C})$, is defined to be the arc number of $f_{\mathcal{C}}$. Two $m$-chord diagrams $\mathcal{C}_{1}=\left(P_{1}, \varphi_{1}\right)$ and $\mathcal{C}_{2}=\left(P_{2}, \varphi_{2}\right)$ are equivalent if there is an orientation-preserving self-homeomorphism $h$ of $\mathbb{S}^{1}$ such that $h\left(P_{1}\right)=P_{2}$ and $h \circ \varphi_{1}=\varphi_{2} \circ h$. From now on we consider $m$-chord diagrams up to this equivalence relation. In the following we sometimes express an $m$-chord diagram $\mathcal{C}=(P, \varphi)$ by $m$ line segments in the plane $\mathbb{R}^{2}$ where $\mathbb{S}^{1} \subset \mathbb{R}^{2}, P$ is the set of the end points of these line segments and $x$ and $\varphi(x)$ are joined by a line segment for each $x \in P$. Thus a line segment expresses a chord, and from now on we do not distinguish them. See for example Figure 1.1.

Let $f: \mathbb{S}^{1} \rightarrow X$ be a map whose multiple points are only finitely many double points. By $\mathcal{C}(f)$ we denote the $c(f)$-chord diagram $\left(\mathrm{S}(f), \varphi_{f}\right)$, where $\varphi_{f}: \mathrm{S}(f) \rightarrow$ $\mathrm{S}(f)$ is the fixed point free involution with $\left.f\right|_{\mathrm{S}(f)} \circ \varphi_{f}=\left.f\right|_{\mathrm{S}(f)}$. We call $\mathcal{C}(f)$ the associated chord diagram of $f$. Then it is clear that $\operatorname{arc}(f)=\operatorname{arc}(\mathcal{C}(f))$.

A chord diagram $\mathcal{D}=(Q, \psi)$ is called a sub-chord diagram of a chord diagram $\mathcal{C}=(P, \varphi)$ if $Q$ is a subset of $P$ and $\psi$ is the restriction of $\varphi$ on $Q$. Then it is clear that $\operatorname{arc}(\mathcal{D}) \leq \operatorname{arc}(\mathcal{C})$. We call $\mathcal{D}=(Q, \psi)$ a proper sub-chord diagram of $\mathcal{C}=(P, \varphi)$ if $\mathcal{D}$ is a sub-chord diagram of $\mathcal{C}$ and $Q$ is a proper subset of $P$.

Let $n$ be a natural number. Let $\mathcal{C}_{2 n+1}$ be a $(2 n+1)$-chord diagram as illustrated in Figure 1.1. Formally $\mathcal{C}_{2 n+1}$ is defined as follows. Let $k$ be a natural number greater than two. Let $R_{k}$ be a regular $k$-gon inscribed in $\mathbb{S}^{1}$ and let $v_{k ; 1}, \cdots, v_{k ; k}$ be the vertices of $R_{k}$ that are arranged in this order on $\mathbb{S}^{1}$ along the counterclockwise orientation of $\mathbb{S}^{1}$; namely, $v_{k ; i}$ and $v_{k ; i+1}$ are adjacent in $R_{k}$ for each $i$, where the indices are considered modulo $k$. Let $j$ be a natural number less than $\frac{k}{2}$. Let $c(k ; i, j)$ be the chord joining $v_{k ; i}$ and $v_{k ; i+j}$ for each $i \in\{1, \cdots, k\}$. Then $\mathcal{C}_{2 n+1}$ is the chord diagram represented by chords $c(4 n+2 ; 2 i-1,2 n-1)$ with $i \in\{1, \cdots, 2 n+1\}$. We will show that $\operatorname{arc}\left(\mathcal{C}_{2 n+1}\right)=3$ but $\operatorname{arc}(\mathcal{D})=2$ for any proper sub-chord diagram $\mathcal{D}$ of $\mathcal{C}_{2 n+1}$. Moreover we have the following theorem.

Theorem 1-1. Let $m$ be a natural number and $\mathcal{C}$ an $m$-chord diagram on $\mathbb{S}^{1}$. Then $\operatorname{arc}(\mathcal{C})=2$ if and only if no sub-chord diagram of $\mathcal{C}$ is equivalent to the chord diagram $\mathcal{C}_{2 n+1}$ for any natural number $n$.

We will give the proof of Theorem 1-1 in Section 2. We note here that after putting the first version of this paper on arXiv, T. Hagge generalized Theorem 1-1 in 2. His theorem characterizes the chord diagrams with arc number $k$ for each natural number $k$.

The motive for this paper was the result in 3 that every knot has a diagram which can be divided into two simple arcs. This result was rediscovered by Ozawa [4] and Shinjo [5]. See also [1]. Then it is natural to ask what plane closed curve can be divided into two simple arcs. Theorem 1-1 gives an answer to this question. However we still have a question whether or not we actually need all of $\mathcal{C}_{3}, \mathcal{C}_{5}, \cdots$. The following proposition answers this question and says that we actually need all of them. 
Proposition 1-2. For each natural number $n$ there exists a smooth immersion $f_{2 n+1}: \mathbb{S}^{1} \rightarrow \mathbb{R}^{2}$ with $\operatorname{arc}\left(f_{2 n+1}\right)=3$ that has only finitely many transversal double points such that the associated chord diagram $\mathcal{C}\left(f_{2 n+1}\right)$ of $f_{2 n+1}$ has a sub-chord diagram which is equivalent to $\mathcal{C}_{2 n+1}$ but has no sub-chord diagram which is equivalent to $\mathcal{C}_{2 m+1}$ for any $m<n$.

We will give the proof of Proposition 1-2 in Section 3.

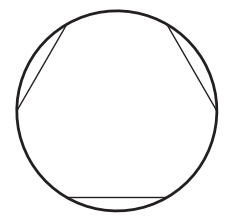

$\mathcal{C}_{3}$

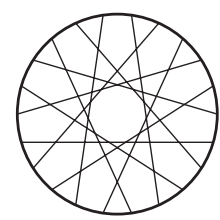

$\mathcal{C}_{11}$

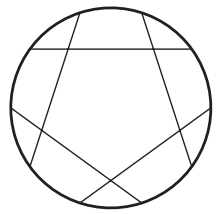

$\mathcal{C}_{5}$

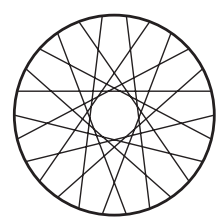

$\mathcal{C}_{13}$

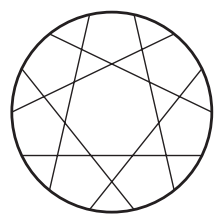

$\mathcal{C}_{7}$

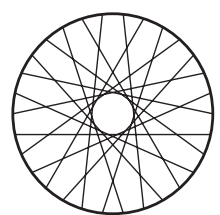

$\mathcal{C}_{15}$

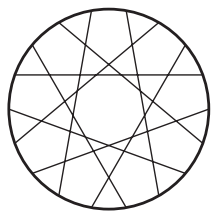

$\mathcal{C}_{9}$

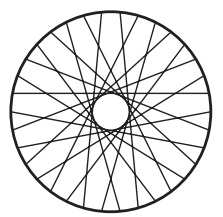

$\mathcal{C}_{17}$

FiguRe 1.1

\section{Proof of Theorem 1-1}

First we check that $\operatorname{arc}\left(\mathcal{C}_{2 n+1}\right)=3$. Let $\mathcal{C}=(P, \varphi)$ be an $m$-chord diagram with $\operatorname{arc}(\mathcal{C})=2$. A pair of points $p, q \in \mathbb{S}^{1} \backslash P$ is called a cutting pair of $\mathcal{C}$ if $x$ and $\varphi(x)$ belong to the different components of $\mathbb{S}^{1} \backslash\{p, q\}$ for each $x \in P$. Then $p$ and $q$ are "antipodal"; namely, each component of $\mathbb{S}^{1} \backslash\{p, q\}$ contains exactly $m$ points of $P$. Thus we can check whether or not a given $m$-chord diagram has arc number 2 by examining $m$ pairs of antipodal points of it. Then by the symmetry of $\mathcal{C}_{2 n+1}$ we immediately have the inequality $\operatorname{arc}\left(\mathcal{C}_{2 n+1}\right)>2$. Then it is easily seen that $\operatorname{arc}\left(\mathcal{C}_{2 n+1}\right)=3$ and $\operatorname{arc}(\mathcal{D})=2$ for any proper sub-chord diagram $\mathcal{D}$ of $\mathcal{C}_{2 n+1}$. The 'only if part' of the proof of Theorem 1-1 immediately follows. The 'if part' immediately follows from the following proposition.

Proposition 2-1. Let $\mathcal{C}$ be a chord diagram on $\mathbb{S}^{1}$ that satisfies the following condition $(*)$ :

$(*) \operatorname{arc}(\mathcal{C}) \geq 3$ and $\operatorname{arc}(\mathcal{D})=2$ for any proper sub-chord diagram $\mathcal{D}$ of $\mathcal{C}$.

Then there is a natural number $n$ such that $\mathcal{C}$ is equivalent to $\mathcal{C}_{2 n+1}$.

Note that deleting a chord will decrease the arc number by at most one. Therefore, if $\mathcal{C}$ is a chord diagram on $\mathbb{S}^{1}$ that satisfies the condition $(*)$, then $\operatorname{arc}(\mathcal{C})=3$. For the proof of Proposition 2-1 we prepare the following lemmas. Let $\mathcal{C}=(P, \varphi)$ be a chord diagram and $c=(x, \varphi(x))$ a chord of $\mathcal{C}$. Let $\alpha$ and $\beta$ be the components of $\mathbb{S}^{1} \backslash\{x, \varphi(x)\}$. We may suppose without loss of generality that $|\alpha \cap P| \leq|\beta \cap P|$. 
Then the length of $c$ in $\mathcal{C}$, denoted by $l(c)=l(c, \mathcal{C})$, is defined to be $|\alpha \cap P|+1$. By $\mathcal{C} \backslash c$, we denote the chord diagram $\left(P \backslash\{x, \varphi(x)\},\left.\varphi\right|_{P \backslash\{x, \varphi(x)\}}\right)$. Let $p, q, x$ and $y$ be four mutually distinct points on $\mathbb{S}^{1}$. We say that the pair of points $p$ and $q$ separates the pair of points $x$ and $y$ if each component of $\mathbb{S}^{1} \backslash\{p, q\}$ contains exactly one of $x$ and $y$. Note that the pair of points $p$ and $q$ separates the pair of points $x$ and $y$ if and only if the chord joining $p$ and $q$ intersects the chord joining $x$ and $y$.

Lemma 2-2. Let $\mathcal{C}=(P, \varphi)$ be a chord diagram on $\mathbb{S}^{1}$ that satisfies the condition $(*)$. Let $c=(x, \varphi(x))$ be a chord of $\mathcal{C}$. Let $p$ and $q$ be a cutting pair of $\mathcal{C} \backslash c$. Then $p$ and $q$ do not separate $x$ and $\varphi(x)$.

Proof. If $p$ and $q$ separate $x$ and $\varphi(x)$, then $p$ and $q$ form a cutting pair of $\mathcal{C}$ itself. Then it follows that $\operatorname{arc}(\mathcal{C})=2$. This is a contradiction.

Lemma 2-3. Let $\mathcal{C}=(P, \varphi)$ be an $m$-chord diagram on $\mathbb{S}^{1}$ that satisfies the condition $(*)$. Let $c=(x, \varphi(x))$ be a chord of $\mathcal{C}$. Then $l(c, \mathcal{C}) \leq m-2$.

Proof. Since $|P|=2 m$ we have $1 \leq l(c, \mathcal{C}) \leq m$ for any chord $c$. First we examine the case $l(c, \mathcal{C})=m$. In this case each component of $\mathbb{S}^{1} \backslash\{x, \varphi(x)\}$ contains exactly $m-1$ elements of $P$. Let $p$ and $q$ be a cutting pair of $\mathcal{C} \backslash c$. Then Lemma 2-2 implies that $p$ and $q$ do not separate $x$ and $\varphi(x)$. Note that each component of $\mathbb{S}^{1} \backslash\{p, q\}$ also contains exactly $m-1$ elements of $P \backslash\{x, \varphi(x)\}$. Then it follows that $p$ and $q$ are next to $x$ and $\varphi(x)$ or $\varphi(x)$ and $x$, respectively. We may suppose without loss of generality that $p$ and $q$ are next to $x$ and $\varphi(x)$, respectively. Let $p^{\prime}$ be a point on $\mathbb{S}^{1}$ that is next to $x$ such that $p^{\prime}$ and $p$ separate $x$ and $\varphi(x)$. Then $p^{\prime}$ and $q$ would be a cutting pair of $\mathcal{C}$. This is a contradiction. Next we examine the case $l(c, \mathcal{C})=m-1$. In this case one component of $\mathbb{S}^{1} \backslash\{x, \varphi(x)\}$ contains exactly $m-2$ elements of $P$, and the other component contains exactly $m$ elements of $P$. Let $p$ and $q$ be a cutting pair of $\mathcal{C} \backslash c$. Then Lemma 2-2 implies that $p$ and $q$ do not separate $x$ and $\varphi(x)$. Note that each component of $\mathbb{S}^{1} \backslash\{p, q\}$ contains exactly $m-1$ elements of $P \backslash\{x, \varphi(x)\}$. Then it follows that one of $p$ and $q$, say $p$, is next to $x$ or $\varphi(x)$, say $x$. Let $p^{\prime}$ be a point on $\mathbb{S}^{1}$ that is next to $x$ such that $p^{\prime}$ and $p$ separate $x$ and $\varphi(x)$. Then $p^{\prime}$ and $q$ would be a cutting pair of $\mathcal{C}$. This is a contradiction. Thus we have $l(c, \mathcal{C}) \leq m-2$.

Lemma 2-4. Let $\mathcal{C}=(P, \varphi)$ be an m-chord diagram on $\mathbb{S}^{1}$ that satisfies the condition $(*)$. Let $c=(x, \varphi(x))$ be a chord of $\mathcal{C}$. Then $l(c, \mathcal{C}) \geq m-2$.

Proof. Suppose that there is a chord $c=(x, \varphi(x))$ of $\mathcal{C}$ with $l(c, \mathcal{C}) \leq m-3$. Let $\mathcal{D}=\left(Q,\left.\varphi\right|_{Q}\right)$ be the sub-chord diagram of $\mathcal{C}$ obtained by deleting all the chords that have at least one end point on the component of $\mathbb{S}^{1} \backslash\{x, \varphi(x)\}$ that has exactly $l(c, \mathcal{C})-1$ points of $P$. Then $l(c, \mathcal{D})=1$. Let $n$ be the number of chords of $\mathcal{D}$. Let $A$ (resp. $B$ ) be the point in $Q$ such that each component of $\mathbb{S}^{1} \backslash\{x, A\}$ (resp. $\left.\mathbb{S}^{1} \backslash\{\varphi(x), B\}\right)$ contains $n-1$ points of $Q$. Note that $n \geq m-(l(c, \mathcal{C})-1) \geq$ $m-(m-3-1)=4$. Therefore $\mathcal{D}$ has at least 4 chords. Then there is a chord $d=(y, \varphi(y))$ of $\mathcal{D}$ such that $\{y, \varphi(y)\}$ and $\{x, \varphi(x), A, B\}$ are mutually disjoint. Suppose that $y$ and $\varphi(y)$ do not separate $x$ and $A$. In this case there must be a chord $e=(z, \varphi(z))$ of $\mathcal{D}$ such that $\{z, \varphi(z)\}$ and $\{x, \varphi(x), y, \varphi(y)\}$ are mutually disjoint and $z$ and $\varphi(z)$ do not separate $y$ and $\varphi(y)$. Then the chords $c, d$ and $e$ form a sub-chord diagram of $\mathcal{C}$ that is equivalent to $\mathcal{C}_{3}$. This is a contradiction. Suppose that $y$ and $\varphi(y)$ separate $x$ and $A$. Let $p$ and $q$ be a cutting pair of $\mathcal{C} \backslash d$. Then we have by Lemma 2-2 that $p$ and $q$ do not separate $y$ and $\varphi(y)$. Note that $p$ 
and $q$ also become a cutting pair of $\mathcal{D} \backslash d$ and they separate $x$ and $\varphi(x)$. Then the component of $\mathbb{S}^{1} \backslash\{p, q\}$ that contains both $A$ and $B$ has more points of $Q \backslash\{y, \varphi(y)\}$ than the other. This is a contradiction.

Thus we have shown the following lemma.

Lemma 2-5. Let $\mathcal{C}=(P, \varphi)$ be an $m$-chord diagram on $\mathbb{S}^{1}$ that satisfies the condition $(*)$. Then $\mathcal{C}$ satisfies the following condition $(\star)$.

$(\star) l(c, \mathcal{C})=m-2$ for every chord $c$ of $\mathcal{C}$.

Proposition 2-6. Let $\mathcal{C}=(P, \varphi)$ be an $m$-chord diagram on $\mathbb{S}^{1}$ that satisfies the condition $(\star)$. If $m$ is even, then $m$ is divisible by 4 and $\operatorname{arc}(\mathcal{C})=2$. If $m$ is odd, then $\mathcal{C}$ is equivalent to $\mathcal{C}_{m}$.

Proof. Recall that $R_{2 m}$ is a regular $(2 m)$-gon inscribed in $\mathbb{S}^{1}$ and $v_{2 m ; 1}, \cdots, v_{2 m ; 2 m}$ are the vertices of $R_{2 m}$ lying in this order. Let $G_{2 m, m-2}$ be the graph whose vertices are $v_{2 m ; 1}, \cdots, v_{2 m ; 2 m}$ and whose edges are the chords $c(2 m ; i, m-2)$ joining the vertices $v_{2 m ; i}$ and $v_{2 m ; i+m-2}$ where $i \in\{1, \cdots, 2 m\}$. By calculating the greatest common divisor $(2 m, m-2)=(2 m-2(m-2), m-2)=(4, m-2)$ we have the isomorphism type of the graph $G_{2 m, m-2}$ as follows.

(1) If $m$ is a multiple of 4 , then $(4, m-2)=2$ and therefore $G_{2 m, m-2}$ is isomorphic to a disjoint union of two $m$-cycles.

(2) If $m$ is congruent to 2 modulo 4 , then $(4, m-2)=4$ and therefore $G_{2 m, m-2}$ is isomorphic to a disjoint union of four $\frac{m}{2}$-cycles.

(3) If $m$ is odd, then $(4, m-2)=1$ and therefore $G_{2 m, m-2}$ is isomorphic to a $2 m$-cycle.

Note that in each case $\mathcal{C}$ must be a perfect matching of the graph $G_{2 m, m-2}$. In (1), up to symmetry, $\mathcal{C}$ is as illustrated in Figure 2.1 Then we have $\operatorname{arc}(\mathcal{C})=2$. In $(2)$, $G_{2 m, m-2}$ has no perfect matchings because an $\frac{m}{2}$-cycle is an odd cycle. In (3), $\mathcal{C}$ is equivalent to $\mathcal{C}_{m}$. This completes the proof.

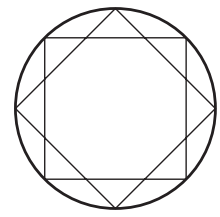

$G_{8,2}$

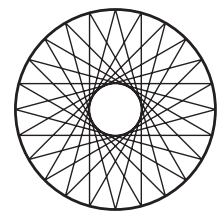

$G_{24,10}$

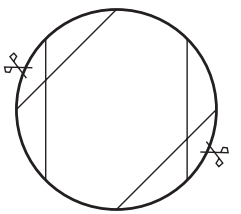

$\mathcal{C}$

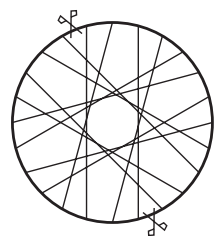

$\mathcal{C}$

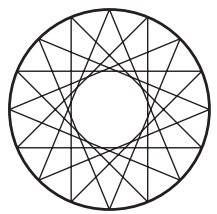

$G_{16,6}$

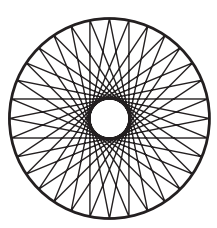

$G_{32,14}$

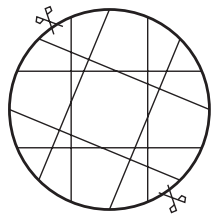

$\mathcal{C}$

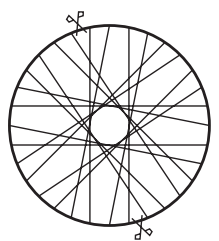

$\mathcal{C}$

FiguRe 2.1

Proof of Proposition 2-1. By Lemma 2-5] and Proposition 2-6 we have the result. 


\section{EXAMPLES OF PLANE CURVES}

Let $\mathcal{C}=(P, \varphi)$ be a chord diagram and let $c=(x, \varphi(x))$ and $d=(y, \varphi(y))$ be two chords of $\mathcal{C}$. We say that $c$ and $d$ are parallel if the pair of points $x$ and $\varphi(x)$ does not separate the pair of points $y$ and $\varphi(y)$. We say that two distinct points $x$ and $y$ in $P$ are next to each other if there is a component of $\mathbb{S}^{1} \backslash\{x, y\}$ that is disjoint from $P$. We say that $c$ and $d$ are close to each other if $x$ and $y$ are next to each other and $\varphi(x)$ and $\varphi(y)$ are next to each other, or $x$ and $\varphi(y)$ are next to each other and $\varphi(x)$ and $y$ are next to each other.

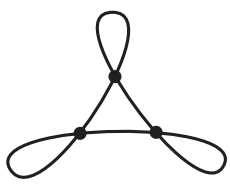

$f_{3}\left(\mathbb{S}^{1}\right)$

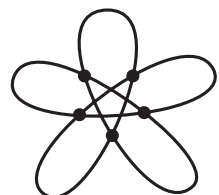

$f_{5}\left(\mathbb{S}^{1}\right)$

FiguRE 3.1

Proof of Proposition 1-2, The cases $n=1,2$ are shown in Figure 3.1. We consider the case $n \geq 3$. Let $G_{2 n+1}=\mathbb{S}^{1} / \sim_{\mathcal{C}_{2 n+1}}$ be the 4-regular graph obtained from $\mathbb{S}^{1}$ by identifying the end points of each chord of $\mathcal{C}_{2 n+1}$. It is easy to observe that $G_{2 n+1}$ is isomorphic to a graph obtained from a $(2 n+1)$-cycle $\Gamma_{2 n+1}$ on vertices $v_{1}, \cdots, v_{2 n+1}$ lying in this order by adding edges joining $v_{i}$ and $v_{i+3}$ for each $i$, so that along the counterclockwise orientation of $\mathbb{S}^{1}$ the vertices of $G_{2 n+1}$ appear as $v_{i}, v_{i+1}, v_{i+1-3}, v_{i+1-3+1}, v_{i+1-3+1-3}, \cdots$. Here the indices of the vertices are listed modulo $2 n+1$. See Figure 3.2. We may suppose that Figure 3.2 illustrates not

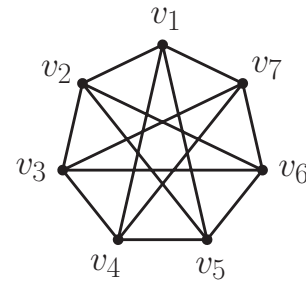

$G_{7}$

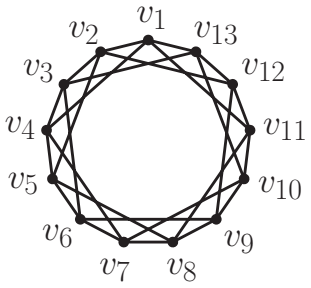

$G_{13}$
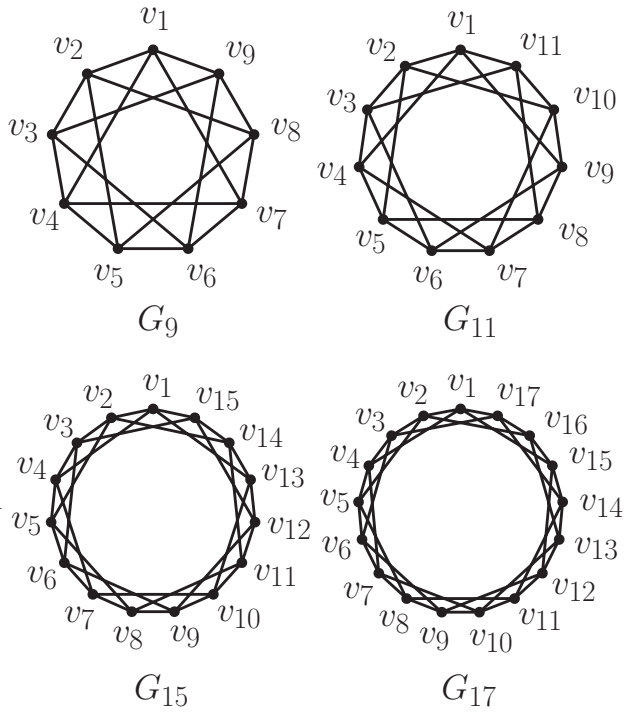

FIGURE 3.2 

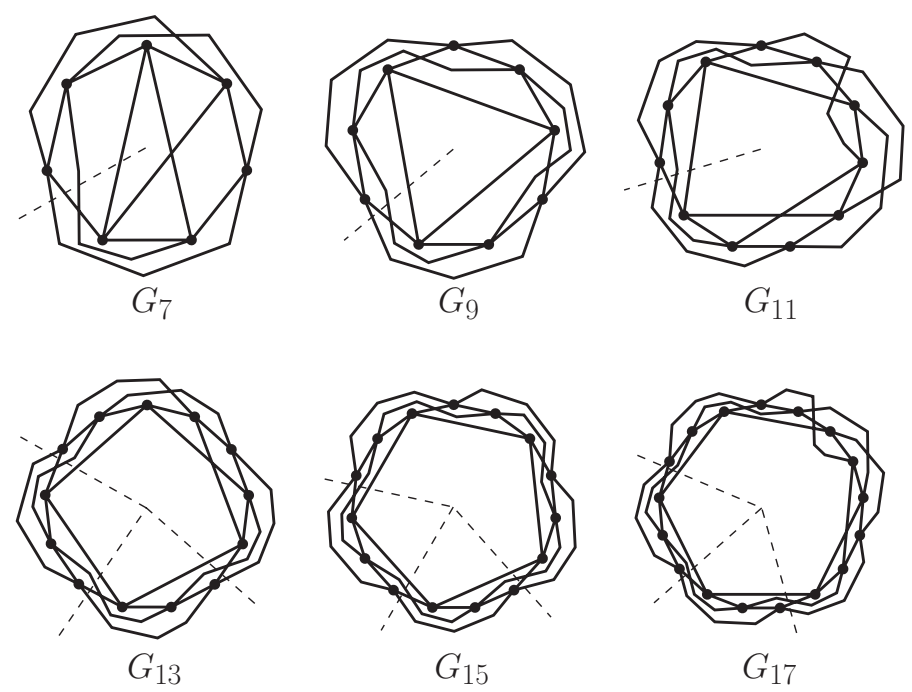

FiguRE 3.3

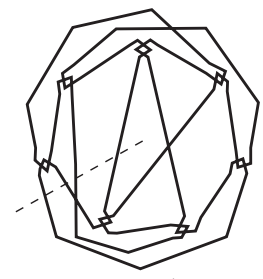

$f_{7}\left(\mathbb{S}^{1}\right)$

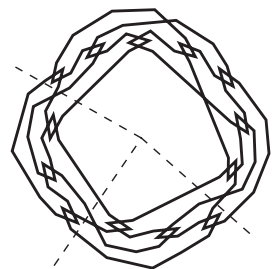

$f_{13}\left(\mathbb{S}^{1}\right)$

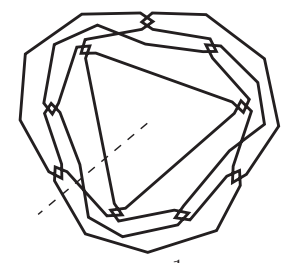

$f_{9}\left(\mathbb{S}^{1}\right)$

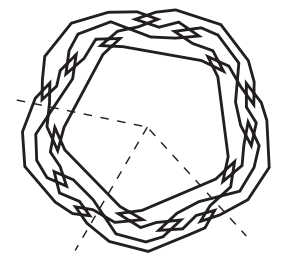

$f_{15}\left(\mathbb{S}^{1}\right)$
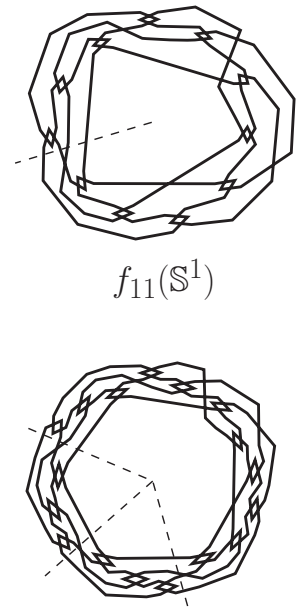

$f_{17}\left(\mathbb{S}^{1}\right)$

Figure 3.4

only abstract graphs but graphs immersed in $\mathbb{R}^{2}$. Then we deform them on $\mathbb{R}^{2}$ as illustrated in Figure 3.3 . Note that they are classified into three types by $2 n+1$ modulo 6 ; namely, $G_{2 n+1+6}$ is obtained from $G_{2 n+1}$ by cutting open $G_{2 n+1}$ along the dotted line and inserting two pieces of a pattern as illustrated in Figure 3.3. We modify this $G_{2 n+1}$ and have the image $f_{2 n+1}\left(\mathbb{S}^{1}\right)$ as illustrated in Figure 3.4. Note that each vertex of $G_{2 n+1}$ is replaced by two transversal double points. We call them a twin pair. The chords corresponding to them are also called a twin pair. By choosing any one of them for each twin pair we have a sub-chord diagram of $\mathcal{C}\left(f_{2 n+1}\right)$ that is equivalent to $\mathcal{C}_{2 n+1}$ by the construction. Observe that each 
$f_{2 n+1}\left(\mathbb{S}^{1}\right)$ is made of $(2 n+1)$-times repetitions of "one step forward and three steps back" along the $(2 n+1)$-cycle $\Gamma_{2 n+1}$ and it totally goes around $\Gamma_{2 n+1}$ twice. Here "one step forward" corresponds to an edge of $G_{2 n+1}$ joining $v_{i}$ and $v_{i+1}$ and "three steps back" corresponds to an edge of $G_{2 n+1}$ joining $v_{i+1}$ and $v_{i+1-3}$. It has no local double points, and each double point comes from a part and another part that is one lap behind. Therefore we have $\operatorname{arc}\left(f_{2 n+1}\right)=3$.

Now we will check that no sub-chord diagram of $\mathcal{C}\left(f_{2 n+1}\right)$ is equivalent to $\mathcal{C}_{2 m+1}$ for any $m<n$. Note that two chords in a twin pair are close to each other in $\mathcal{C}\left(f_{2 n+1}\right)$. Let $\mathcal{D}\left(f_{2 n+1}\right)$ be a sub-chord diagram of $\mathcal{C}\left(f_{2 n+1}\right)$ obtained from $\mathcal{C}\left(f_{2 n+1}\right)$ by deleting one of two chords for each twin pair in $\mathcal{C}\left(f_{2 n+1}\right)$. Since no two chords in $\mathcal{C}_{2 m+1}$ are close to each other it is sufficient to check that no sub-chord diagram of $\mathcal{D}\left(f_{2 n+1}\right)$ is equivalent to $\mathcal{C}_{2 m+1}$ for any $m<n$. Suppose that $\mathcal{E}$ is a sub-chord diagram of $\mathcal{D}\left(f_{2 n+1}\right)$ that is equivalent to $\mathcal{C}_{2 m+1}$ for some $m<n$. Since no proper sub-chord diagram of $\mathcal{C}_{2 n+1}$ is equivalent to $\mathcal{C}_{2 m+1}$, there is a chord $c$ of $\mathcal{E}$ that does not belong to any twin pair of $\mathcal{C}\left(f_{2 n+1}\right)$; namely, $c$ corresponds to a transversal double point of $f_{n}$ that comes from a double point of $G_{2 n+1} \subset \mathbb{R}^{2}$ in Figure 3.3 . Observe that for each chord $d=(x, \varphi(x))$ of $\mathcal{C}_{2 m+1}$ there exist exactly two chords $g=(y, \varphi(y))$ and $h=(z, \varphi(z))$ of $\mathcal{C}_{2 m+1}$ that are parallel to $d$ such that all of $y, \varphi(y), z$ and $\varphi(z)$ are contained in the same component of $\mathbb{S}^{1} \backslash\{x, \varphi(x)\}$.

Therefore $c$ must have such two chords in $\mathcal{D}\left(f_{2 n+1}\right)$. By the "one step forward and three steps back" structure of $f_{2 n+1}\left(\mathbb{S}^{1}\right)$ mentioned above the double points corresponding to such chords must lie in a small neighbourhood of the double point corresponding to $c$. Then we can check that there are no such two chords for $c$ in $\mathcal{D}\left(f_{2 n+1}\right)$ except that the case $2 n+1$ is congruent to 5 modulo 6 and $c$ is one of the three chords of $\mathcal{C}\left(f_{2 n+1}\right)$ that come from the three double points on the same edge of $G_{2 n+1} \subset \mathbb{R}^{2}$ in Figure 3.3 .

For this exceptional case we further observe that the chords $g$ and $h$ above intersect unless $m=1$ and the end point of $g$ (resp. $h$ ) that is next to $x$ or $\varphi(x)$ in $\mathcal{C}_{2 m+1}$ is not next to any end point of $h$ (resp. $g$ ) in $\mathcal{C}_{2 m+1}$.

However in this exceptional case we can check that there are no such two chords for $c$ in $\mathcal{D}\left(f_{2 n+1}\right)$. This is a contradiction.
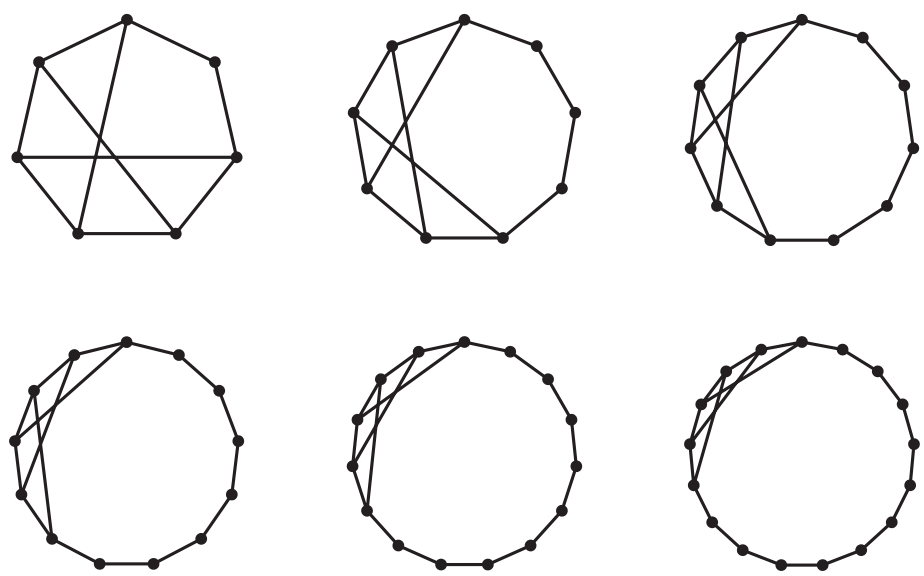

FiguRE 3.5 
Remark 3-1. It is easy to see that the graph $G_{2 n+1}=\mathbb{S}^{1} / \sim_{\mathcal{C}_{2 n+1}}$ is a non-planar graph for $n \geq 2$. In fact $G_{5}$ is the complete graph $K_{5}$, and $G_{2 n+1}$ with $n \geq 3$ contains a subgraph that is a subdivision of the complete bipartite graph $K_{3,3}$. See Figure 3.5. Therefore for $n \geq 2$ there is no smooth immersion $f: \mathbb{S}^{1} \rightarrow \mathbb{R}^{2}$ that has only finitely many transversal double points whose associated chord diagram $\mathcal{C}(f)$ itself is equivalent to $\mathcal{C}_{2 n+1}$.

\section{ACKNOWLEDGMENTS}

This work was done while the author was visiting George Washington University. The author is grateful for the hospitality of the mathematical department of George Washington University. The author is thankful to Professor Józef Przytycki, Dr. Ken Shoda and Dr. Radmila Sazdanović for valuable discussions on mathematics. The author is also grateful to the referee, whose comments improved the presentation of the paper.

\section{REFERENCES}

[1] C. Adams, R. Shinjo and K. Tanaka, Complementary regions of knot and link diagrams, arXiv:0812.2558 (2008).

[2] T. Hagge, to appear.

[3] G. Hotz, Arkadenfadendarstellung von Knoten und eine neue Darstellung der Knotengruppe (German), Abh. Math. Sem. Univ. Hamburg, 24 (1960), 132-148. MR0111047(22:1912)

[4] M. Ozawa, Edge number of knots and links, arXiv:0705.4348 (2007).

[5] R. Shinjo, Complementary regions of projections of spatial graphs, in preparation.

Department of Mathematics, School of Education, Waseda University, Nishi-Waseda 1-6-1, SHINJUKu-KU, TOKYO, 169-8050, JAPAN

E-mail address: taniyama@waseda.jp 